\title{
Dramatic effect of thermal mismatch on the structural, dielectric, ferroelectric and pyroelectric properties in low-cost epitaxial PZT films on $\mathrm{SrTiO}_{3}$ and $\mathrm{Si}(001)$ substrates
}

\author{
R. Moalla ${ }^{1}$, B. Vilquin ${ }^{1}$, G. Saint-Girons ${ }^{1}$, G. Sebald ${ }^{2}$, N. Baboux ${ }^{3}$, and R. Bachelet ${ }^{1, a)}$ \\ ${ }^{1}$ Institut des Nanotechnologies de Lyon (INL) - CNRS UMR 5270, Ecole Centrale de Lyon, \\ Bâtiment F7, 36 av. Guy de Collongue, 69134 Ecully Cedex, France \\ ${ }^{2}$ Laboratoire de Génie Electrique et Ferroélectricité (LGEF) - EA 682, INSA de Lyon, Bâtiment \\ Gustave Ferrié, 8 rue de la Physique, 69621 Villeurbanne Cedex, France \\ ${ }^{3}$ Institut des Nanotechnologies de Lyon (INL) - CNRS UMR 5270, INSA de Lyon, Bâtiment \\ Blaise Pascal, 7 avenue Jean Capelle, 69621 Villeurbanne Cedex, France \\ a) Email: romain.bachelet@ec-lyon.fr
}

Different structural orientations, strongly impacting the physical properties, are generated depending on the thermal expansion mismatch between epitaxial $\mathrm{Pb}(\mathrm{Zr}, \mathrm{Ti}) \mathrm{O}_{3}$ film and the substrate. Here, either mainly $c$-oriented or mainly $a$-oriented PZT layers are obtained on $\mathrm{SrTiO}_{3}$ or $\mathrm{Si}(001)$ substrates, respectively. About a factor of two in the resulting dielectric, ferroelectric and pyroelectric properties are probed along the out-of-plane direction. These results are of critical importance to design monolithically integrated devices of enhanced efficiency. 
Because of its inclusive pyroelectric and piezoelectric properties, ferroelectric oxides are of great interest for a wide range of key applications such as memories, transducers, sensors, modulators, actuators and energy harvesters, taking advantage of their ability to manipulate and convert electrical, mechanical, thermal and/or optical physical magnitudes. ${ }^{1,2}$ Especially, pyroelectric properties, are appealing for a wide range of devices such as detectors, imaging tools, biosensors and thermal energy harvesters. ${ }^{3-9}$ However, the structural-pyroelectric properties relationships are much less known that piezoelectric and ferroelectric properties, limiting the progress toward further pyroelectric devices. ${ }^{2,3,9}$

Pyroelectricity evolves from a change of electric polarization with a change of temperature. Since electric polarization is structurally dependent, pyroelectric properties could be enhanced in single-crystalline films with controlled orientation and epitaxial strain, as it is the case for ferroelectric properties. ${ }^{2}$ For instance, enhancement of out-of-plane polarization by in-plane compressive strain has been demonstrated with epitaxial $\mathrm{BaTiO}_{3}$ films on oxide substrates. ${ }^{2}$ Anisotropy of ferroelectric and electro-optical properties have also been shown with the widely studied $\mathrm{Pb}\left(\mathrm{Zr}_{\mathrm{x}} \mathrm{Ti}_{1-\mathrm{x}}\right) \mathrm{O}_{3}$ (PZT) films mainly on $\mathrm{SrTiO}_{3}$ (STO) substrates with different crystallographic orientations. ${ }^{10,11}$ However, it has been scarcely probed within the same main crystallographic orientation. Slight differences of pyroelectric properties have been shown in epitaxial PZT films by changing their epitaxial strain within a reduced range on oxide substrates. ${ }^{12}$ Nevertheless, for practical applications into real devices, ferroelectric oxides should be integrated, preferably by low-cost elaboration techniques, on semiconductor platforms as silicon. ${ }^{13-15}$ The large difference in thermal expansion coefficients (TEC) between oxides and semiconductors can lead to very different strain states in the oxide films that can dramatically 
change its physical properties. For instance, the TEC of STO and PZT is about four times higher than that of $\mathrm{Si}^{16}$

In this communication, we report on the large effects of thermal expansion mismatch between PZT films elaborated by low-cost sol-gel process and key substrates (STO and Si) on the structural, dielectric, ferroelectric and pyroelectric properties. PZT has been used here because it is still a major actor for the above-mentioned wide-range applications due to its large spontaneous polarization, large piezoelectric and pyroelectric coefficients. ${ }^{2,3,4,7-9,17}$ However, the study presented here can be straightforwardly extended to lead-free epitaxial ferroelectric oxides.

Epitaxial PZT (52:48) layers (500 nm thick) were grown by the same sol-gel process on $30 \mathrm{~nm}$ thick $\mathrm{SrRuO}_{3}(\mathrm{SRO})$ layers epitaxially grown by radio frequency (rf) magnetron sputtering at $800^{\circ} \mathrm{C}$ under $510^{-3}$ mbar of partial oxygen pressure on both (001)-oriented single-crystalline STO substrate and Si substrate buffered with $\sim 10 \mathrm{~nm}$ thick epitaxial STO layers grown by oxide molecular beam epitaxy (MBE). ${ }^{18,19}$ The SRO layers are used as lattice-matched bottom electrodes in the built-in metal-insulator-metal (MIM) capacitor epitaxial heterostructures. By sol-gel process, each step including spin-coating and crystallization by rapid thermal annealing at $650^{\circ} \mathrm{C}$ under oxygen leads to the formation of a $33 \mathrm{~nm}$ thick PZT layer so that 15 cycles were performed to reach the desired PZT thickness of $\sim 500 \mathrm{~nm} \cdot{ }^{20,21}$ Atomic force microscopy (AFM) topographic analyses in tapping mode show a flat surface with root-mean-square roughness less than $0.7 \mathrm{~nm}$ for both heterostructures (Fig. 1). High brilliance X-ray diffractometer (XRD, Rigaku SmartLab equipped with a $9 \mathrm{~kW}$ rotating anode and a two-bounce Ge(220) monochromator) was used to investigate the crystalline orientation and the structure of the layers. After the PZT growth, Pt was deposited by sputtering at room temperature and square- 
shaped top electrodes were made using a UV-lithography and lift-off process for further macroscopic electrical characterizations. The pyroelectric properties were determined by measuring ferroelectric hysteresis loops (polarization versus electric field) at different stabilized temperatures from $25^{\circ} \mathrm{C}$ to $125^{\circ} \mathrm{C}$.

The out-of-plane XRD 29/ $\omega$ scans recorded on both samples are displayed in Figure 2. From these scans and in-plane scans (not shown here), the epitaxial relationship between PZT and the substrates have been deduced and reads, as previously reported: $[100] \operatorname{PZT}(001) / /[100] \operatorname{SRO}(001) / /[100] \operatorname{STO}(001) / /[110] \operatorname{Si}(001) .{ }^{20,21}$ Both films appear singlecrystalline with a $\{001\}$-orientation. However, the PZT $\{002\}$ Bragg peak is either positioned close to (200) or (002) tetragonal reflection, corresponding to $a$ - or $c$-axis orientation, respectively. Out-of-plane elongated lattice (c-orientation) seems to dominate in the PZT layer grown on STO (Fig. 2(a)) whereas in-plane elongated lattice ( $a$-orientation) seems to dominate on Si substrate (Fig. 2(b)). The corresponding out-of-plane lattice parameters are $4.12 \AA$ and $4.048 \AA$ on STO and Si substrate, respectively. Reciprocal space maps around the PZT (103) node were carried out to measure the in-plane lattice parameters and further investigate the structure of the PZT layer in more details for both cases (Fig. 3(a-b)). In agreement with the 20/ scans, the structural properties of the PZT layers are confirmed to be clearly different depending on the substrate used. Mostly $c$-axis oriented PZT layers are obtained on STO substrate (Fig. 3(a)), whereas $a$-axis oriented PZT layers are obtained on Si substrate (Fig. 3(b)), although a small amount of rhombohedral phase may exist in the layer. The averaged in-plane lattice parameters are $4.098 \AA$ for PZT on STO substrate, and $4.10 \AA$ for PZT on Si substrate, giving an out-of-plane over in-plane lattice parameters ratio of 1.005 and 0.987 for PZT on STO and Si, 
respectively (i.e., $+0.5 \%$ out-of-plane elongation and $+1.3 \%$ in-plane elongation with respect to a cubic lattice, on $\mathrm{STO}$ and $\mathrm{Si}$, respectively).

The lattice strain in an epitaxial film can be explained by the lattice mismatch between the film and the bottom layer, the lattice relaxation (elastic relaxation occurring with thickness and plastic relaxation from lattice-misfit accommodation dislocations), and the thermal expansion mismatch between the film and the substrate combined with two-dimensional clamping during cooling the sample down from growth/crystallization temperature through Curie temperature at which the domain structure forms. ${ }^{22}$ Based on previous studies, ${ }^{23}$ the total strain of the film can be defined as follows:

$$
(1-\mathrm{R}) f_{0}+\tau
$$

where $\mathrm{R}$ is the relaxation ratio, $f_{0}$ the epitaxial strain due to the lattice mismatch and $\tau$, the lattice strain due to the thermal expansion mismatch. ${ }^{24}$ The lattice mismatch $f_{0}$ between PZT and the substrates are relatively large (theoretically around $-3.2 \%$ on STO and $-5.2 \%$ on $\mathrm{Si}$ at growth temperature, if the bottom layers are fully strained ${ }^{25}$ ), both leading theoretically to inplane compressive strain favoring out-of-plane domain orientation/elongation. For PZT layers grown on STO substrates, the thermal expansion mismatch is negligible since the thermal expansion coefficients of STO and PZT are similar. ${ }^{16}$ In that case, the PZT film suffers rather from in-plane compressive strain (mainly coming from the lattice mismatch), and preferentially yields $c$-orientation. Whereas, the thermal expansion mismatch is not negligible the PZT layers on $\mathrm{Si}$ and it adds in-plane tensile strain contribution of about $\tau=+0.4 \%{ }^{24}$ Since mainly $a$ orientation is observed in that case, the thermal expansion mismatch seems to dominate on $\mathrm{Si}$ with a lattice relaxation close to $100 \%$. That is consistent with the large lattice mismatches and the large thickness of the PZT layers $(500 \mathrm{~nm})$, in addition to the present elaboration by sol-gel 
process that includes sequential cycles of crystallization annealing and that may enlarge the effect of thermal mismatch. From the $\mathrm{Q}_{\mathrm{x}}$ line profiles centered on the $a$-orientated PZT reflection at $\mathrm{Q}_{\mathrm{z}}=0.742 \AA^{-1}$ on the reciprocal space maps (Figs. 3(c-d)), we can confirm that the PZT layers are most-likely tetragonal (where at least two in-plane components were needed to properly fit the global peak, corresponding to in-plane lattice parameters of $a$-oriented tetragonal PZT layer, about $4.13 \AA$ and $4.08 \AA \AA$ on STO and $4.12 \AA$ and $4.05 \AA \AA$ on $\mathrm{Si}$ ). These results are consistent with theoretical studies, even if a distorted rhombohedral phase component may partly exist in the layers. $^{26}$

Ferroelectric properties of both heterostructures were analyzed along the out-of-plane direction by measuring the electric polarization versus electric field (P-E) at room temperature using PUND (Positive Up Negative Down) excitation pulse trains allowing to discriminate the polarization current from the dielectric displacement and leakage current. ${ }^{27,28}$ Figure 4(a) shows that the out-of-plane remnant polarization $\left(\mathrm{P}_{\mathrm{r}}\right)$ is much larger for the PZT films on STO than that on $\mathrm{Si}\left(22.5 \mu \mathrm{C} / \mathrm{cm}^{2}\right.$ and $13.5 \mu \mathrm{C} / \mathrm{cm}^{2}$, respectively), in agreement with the measured structural orientations. The non-zero out-of-plane polarization in the PZT layer on Si can come from some $c$-oriented tetragonal domains or rhombohedral domains that may exist. The rhombohedral phase, having its spontaneous electric polarization lying along the $<111>$ pseudo cubic unit cell, can be still switchable in vertical MIM stack. ${ }^{29}$ The relative dielectric constant along the out-ofplane direction $\left(\varepsilon_{33 r}\right)$, extracted from capacitance versus electric field measurements $(\mathrm{C}-\mathrm{V})$, is more than twice larger for PZT on Si than on STO substrate (Fig. 4(b)), consistent with the fact that the dielectric constants have maximum values in the directions perpendicular to the spontaneous polarization of PZT. ${ }^{30}$ Values of 490 and 1083 were obtained at zero electric field 
on STO and Si substrates, respectively; and $\varepsilon_{33 r} \sim 165$ on both substrates at high electric field $(800 \mathrm{kV} / \mathrm{cm})$.

In order to extract the corresponding pyroelectric coefficients along the out-of-plane direction, ferroelectric hysteresis loops were recorded for both heterostructures at different sample temperatures ranging from $25{ }^{\circ} \mathrm{C}$ to $125^{\circ} \mathrm{C}$ (Fig. 5(a-b)). As expected, the remnant polarization (after positive electric field switching) decreases when the temperature increases. The variation of $\mathrm{P}_{\mathrm{r}}$ as a function of the temperature is shown for both heterostructures in Fig. 5(c). The pyroelectric coefficients $(p)$ extracted from these measurements are -680 and $-450 \mu \mathrm{C} \cdot \mathrm{m}^{-2} \cdot \mathrm{K}^{-1}$ for PZT on STO and Si substrates, respectively. These coefficients are consistent with those reported in previous studies and the one measured here on $c$-oriented PZT layers is found slightly higher than some measured on polycrystalline PZT films. ${ }^{4,7,9,12,31}$ The difference between these two values here is attributed to the difference in the structural orientation and the corresponding amplitude of the remnant polarization. Again, the non-zero pyroelectric coefficient for PZT on Si must come from some $c$-oriented domains or rhombohedral domains. It is worth to notice that $p$ are almost constant on the whole temperature and electric field range, revealing the absence of decrease of $p$ both under high electric field or large temperature difference, which is consistent to the fact that we are working far from Curie temperature (polarization vs temperature exhibit a quasi-linear behavior). Based on these measurements, the densities of pyroelectric energy conversion $\left(W_{D}\right)$ are estimated by a Synchronized Electric Charge Extraction (SECE) technique, ${ }^{9,32}$ and calculated by equation (2)

$W_{D}=\frac{p^{2}}{\varepsilon_{33}} \times \Delta T^{2}$ 
where $\varepsilon_{33}$ is the dielectric constant along the out-of-plane direction and $\Delta T$ is the temperature change. The converted pyroelectric energy densities, under field as it is the case in practice with harvesting cycles, ${ }^{6,8,9,32}$ are plotted in function of the temperature change in Figure 5(d). They are twice larger for PZT on STO than on Si. For instance for a temperature variation of $\Delta \mathrm{T}=10^{\circ} \mathrm{C}$, they are about 30 and $16 \mathrm{~mJ} . \mathrm{cm}^{-3}$ per thermal cycle, on STO and Si substrates, respectively.

In conclusion, 500-nm-thick epitaxial pyroelectric PZT layers were grown by sol-gel process in MIM capacitor structure on both STO and Si (001) substrates. Thermal expansion mismatch between film and substrate dramatically affects the structural orientations and the consequent physical properties. Due to non negligible thermal mismatch between PZT and $\mathrm{Si}$, the induced tensile strain favors $a$-orientation in the PZT layer, whereas PZT on STO is mainly $c$-oriented. Along the out-of-plane direction, remnant polarization and pyroelectric coefficient are found almost twice larger on PZT layers on STO than on $\mathrm{Si}\left(22.5\right.$ and $13.5 \mu \mathrm{C} / \mathrm{cm}^{2}$; and -680 and -450 $\mu \mathrm{C} / \mathrm{m}^{2} \mathrm{~K}$, respectively) whereas relative dielectric constant of PZT layers is found more than twice larger on Si than on STO (1080 and 490, respectively). The corresponding conversion of pyroelectric energy densities has then been deduced to be larger close to the polarization axis, about twice larger for PZT layers on STO than on Si $\left(30\right.$ and $16 \mathrm{~mJ} / \mathrm{cm}^{3}$, respectively). In addition to the lattice mismatch and its associated epitaxial strain engineering, the thermal expansion mismatch can then be viewed as an efficient tool to control the structural and the induced physical properties. Silicon appears as an interesting substrate for the integration of inplane pyroelectric devices of enhanced energy conversion.

\section{ACKNOWLEDGMENTS}


The authors acknowledge the region Rhône-Alpes for the doctoral fellowship of R. Moalla, the "Cellule Energie" of the Centre National de la Recherche Scientifique (CNRS) for the financial support of the exploratory project "PYROS", and Ecole Centrale de Lyon (ECL) for the funding of emergent thematic and transversal projects. The authors gratefully thank A. Benamrouche and Y. Robach for AFM characterizations, and P. Regreny, C. Botella, J. B. Goure, R. Mazurczyk and P. Cremillieu for technical assistance on the Nanolyon technological platform. 


\section{REFERENCES}

1"Thin Films and Heterostructures for Oxide Electronics", Edited by S. B. Ogale, New York: Springer (2005).

2"Physics of ferroelectrics - A modern perspective", Eds. K. Rabe, Ch. H. Ahn, J.-M. Triscone, Springer-Verlag Berlin Heidelberg (2007).

${ }^{3}$ S. B. Lang, Phys. Today 58, 31 (2005).

${ }^{4}$ S. P. Alpay, J. Mantese, S. Trolier Mc-Kinstry, Q. Zhang, and R. W. Whatmore, MRS Bull. 39, 1099 (2014).

${ }^{5}$ M. S. Heimlich, U. J. Krull, R. F. Debono, and R. S. Brown, In Biosensor technology:

Fundamentals and applications; Eds. R. B. Buck, W. E. Hatfield, M. Umana, E. F. Bowden, M.

Dekker, INC: New York, 1990; chapter 24, pp 337-349.

${ }^{6}$ R. B. Olsen and D. Evans, J. Appl. Phys. 54, 5941 (1983).

${ }^{7}$ R. Whatmore and R. Watton, Ferroelectrics 236, 259 (2000).

${ }^{8}$ G. Sebald, D. Guyomar, and A. Agbossou, Smart Mater. Struct. 18, 125006 (2009).

${ }^{9}$ C. R. Bowen, J. Taylor, E. LeBoulbar, D. Zabek, A. Chauhan, and R. Vaish, Energy Environ.

Sci. 7, 3836 (2014).

${ }^{10}$ K. Nagashima, M. Aratani, H. Funakubo, J. Appl. Phys. 89, 4517 (2001).

${ }^{11}$ M. Zhu, Z. Du, L. Jing, A. L. Y. Tok, E. H. T. Teo, Appl. Phys. Lett. 107, 031907 (2015).

${ }^{12}$ J. Karthik, J. C. Agar, A. R. Damodaran, and L. W. Martin, Phys. Rev. Lett. 109, 257602 (2012).

${ }^{13}$ A. A. Demkov, A. B. Posadas, "Integration of functional oxides with semiconductors", Springer New York (2014). 
${ }^{14}$ J. W. Reiner, A. M. Kolpak, Y. Segal, K. F. Garrity, S. Ismail-Beigi, Ch. A. Ahn, F. J. Walker, Adv. Mat. 22, 2919 (2010).

${ }^{15}$ S-H. Baek and C-B. Eom, Acta Mater. 61, 2734 (2013).

${ }^{16} \mathrm{TEC}$ of Si is about $2.610^{-6} /{ }^{\circ} \mathrm{C}$, see Yim, W.M., and R.J. Paff, J. Appl. Phys. 45, 1456 (1974).

TEC of $\mathrm{SrTiO}_{3}$ is about $910^{-6} /{ }^{\circ} \mathrm{C}$, see the data sheet of CrysTec single-crystals. TEC of PZT is closer to $910^{-6}{ }^{\circ} \mathrm{C}$, see G. Shirane et al., J. Phys. Soc. Jpn. 7, 12 (1952).

17. Y. Yang, S. Wang, Y. Zhang, and Z. L. Wang, Nano Lett. 12, 6408 (2012).

${ }^{18}$ G. Delahaye, C. Merckling, M. El-Kazzi, G. Saint-Girons, M. Gendry, Y. Robach, and G. Hollinger, J. Appl. Phys. 100, 124109 (2006).

${ }^{19}$ G. Niu, G. Saint-Girons, B. Vilquin, G. Delhaye, J.-L. Maurice, C. Botella, Y. Robach, and G. Hollinger, Appl. Phys. Lett. 95, 062902 (2009).

${ }^{20}$ S. Yin, G. Niu, B. Vilquin, B. Gautier, G. Le Rhun, E. Defay, and Y. Robach, Thin Solid Films 520, 4572 (2012).

${ }^{21}$ S. Yin, M. Cueff, G. Niu, G. Le Rhun, B. Vilquin, G. Saint Girons, R. Bachelet, B. Gautier, Y. Robach, P. Gemeiner, N. Guiblin, E. Defay, and B. Dkhil, J. Appl. Phys. 115, 214108 (2014).

${ }^{22}$ R. A. Wolf and S. Trolier-McKinstry, J. Appl. Phys. 95, 1397 (2004).

${ }^{23}$ A. Sambri, S. Gariglio, A. Torres Pardo, J.-M. Triscone, O. Stéphan, J. W. Reiner, and C. H. Ahn, Appl. Phys. Lett. 98, 012903 (2011)

${ }^{24}$ The relaxation ratio $\mathrm{R}$ is defined here as: $\left(a_{/ /}-a_{b}\right) /\left(a_{f}-a_{b}\right)$, where $a_{/ /}$is the measured in-plane lattice parameter of the film, $a_{b}$ the in-plane parameter of the bottom layer and $a_{f}$ the bulk inplane parameter of the layer. The epitaxial strain due to the lattice mismatch $f_{0}$ is defined here as: $\left(a_{b}-a_{f}\right) / a_{f}$, where $a_{f}$ and $a_{b}$ are the in-plane parameters at the growth temperature of the film and the substrate, respectively. And the strain due to the thermal expansion mismatch $\tau$ is defined 
here as: $\left(\alpha_{f}-\alpha_{s}\right) \times\left(T_{g}-T\right)$, where $\alpha_{f}$ is the thermal expansion coefficient of the film, $\alpha_{s}$ the thermal expansion coefficient of the substrate, $T_{g}$ the growth temperature and $T$ the temperature at which the sample is structurally characterized.

${ }^{25}$ Note that each upper layer, especially SRO and $\mathrm{STO}$ on $\mathrm{Si}$, has suffered from strain due to thermal expansion mismatch, as measured here ( $c f$. Fig. 3b). Thus, the lattice mismatch between PZT and SRO on Si is closer to $-2.3 \%$ than $-5.2 \%$.

${ }^{26}$ V. G. Kukhar, N. A. Pertsev, H. Kohlstedt, and R. Waser, Phys. Rev. B 73, 214103 (2006).

${ }^{27}$ J. F. Scott, C. A. Araujo, H. B. Meadows, L. D. McMillan, and A. Shawabkeh, J. Appl. Phys. 66, 1444 (1989).

${ }^{28}$ I. Fina, L. Fábrega, E. Langenberg, X. Martı', F. Sánchez, M. Varela, and J. Fontcuberta, J. Appl. Phys. 109, 074105 (2011).

${ }^{29}$ S. K. Streiffer, C. B. Parker, A. E. Romanov, M. J. Levefre, L. Zhao, J. S. Speck, W. Pompe, C. M. Foster, and G. R. Bai, J. Appl. Phys. 83, 2742 (1998).

${ }^{30}$ X. Du, U. Belegundu, and K. Uchino, Jpn. J. Appl. Phys. 36, 5580 (1997).

${ }^{31}$ R. Moalla, G. Le Rhun, E. Defay, N. Baboux, G. Sebald, R. Bachelet, Thin Sol. Films, in press ${ }^{32}$ G. Sebald, E. Lefeuvre, and D. Guyomar, IEEE Transactions on Ultrasonics, Ferroelectricity and Frequency Control 55, 538 (2008). 


\section{FIGURES \& CAPTIONS}
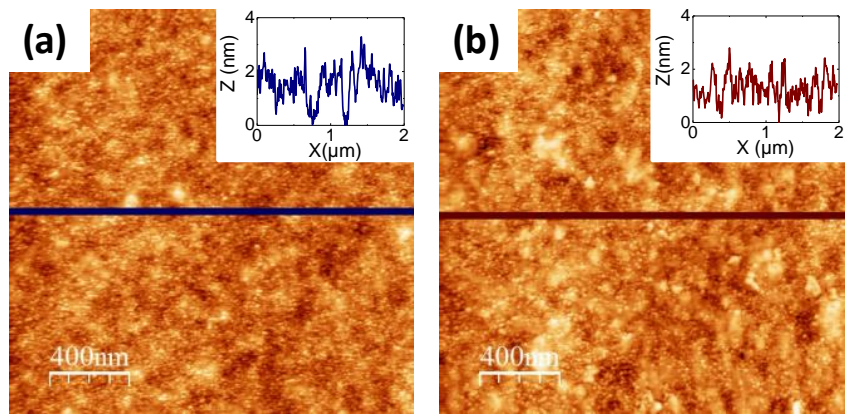

Fig. 1. $2 \times 2 \mu \mathrm{m}^{2}$ AFM topographic images of the complete heterostructures grown on (a) $\mathrm{SrTiO}_{3}(001)$ substrate and (b) STO-buffered $\mathrm{Si}(001)$ substrate, with the corresponding topographic profiles. 


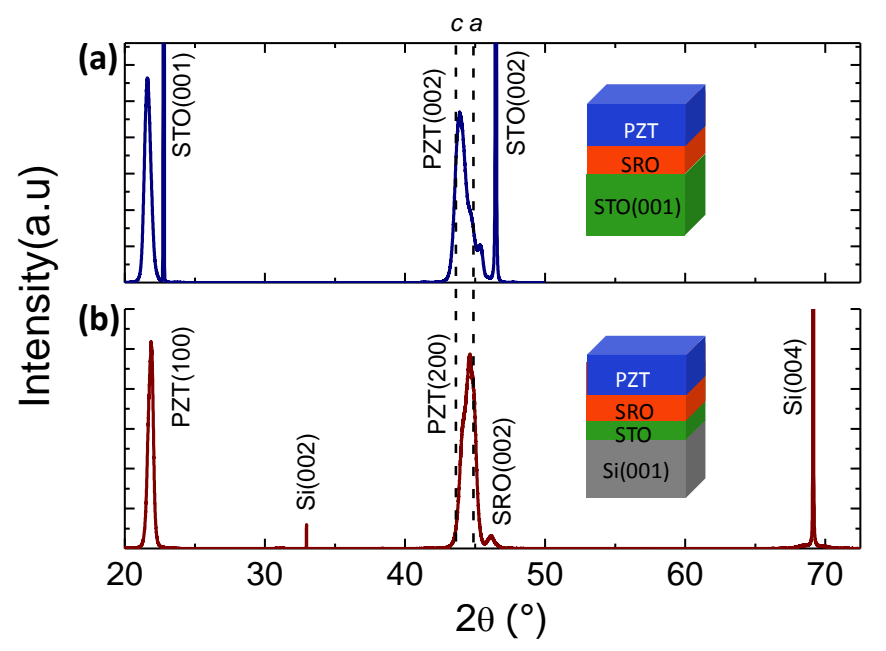

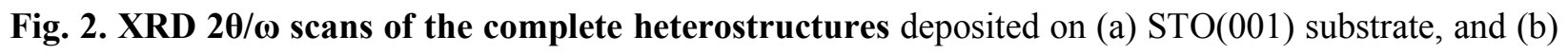
STO-buffered $\operatorname{Si}(001)$ substrate. Dash lines mark the $a$ - and $c$-oriented domains in the $\{002\}$ reflection of bulk tetragonal PZT, as a guide for the eyes. 


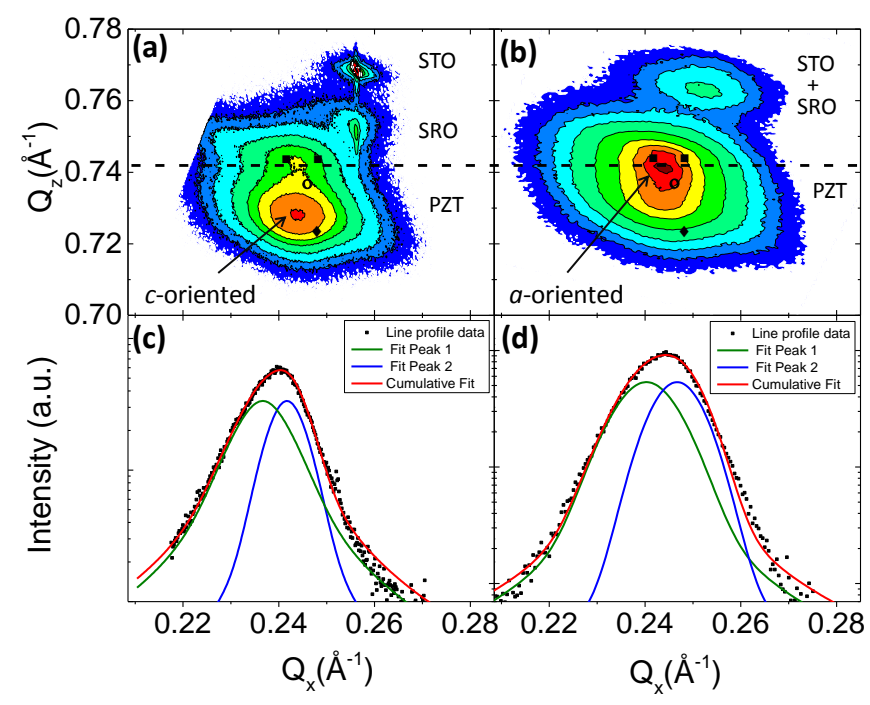

Fig. 3. Reciprocal space maps around the PZT (103) node on (a) PZT/SRO/STO(001) heterostructure, and (b) $\mathrm{PZT} / \mathrm{SRO} / \mathrm{STO} / \mathrm{Si}(001)$ heterostructure. Intensities from low to high: blue, green, yellow, red. As a guide for the eyes, the symbols indicate the bulk (103) reflections of $c(\checkmark), a_{1}$ and $a_{2}(\boldsymbol{\square})$ domains of tetragonal PZT, and of $a$ of the rhombohedral phase $(\circ)$. (c,d) $Q_{x}$ line profiles corresponding to the marked dashed lines in $(a, b)$, respectively, with fits corresponding to the in-plane parameters of the $a_{1}$ and $a_{2}$ domains of the tetragonal phase. 

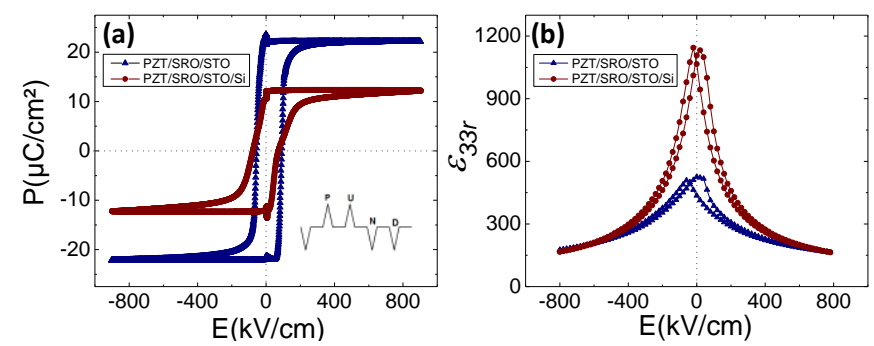

Fig. 4. Out-of-plane ferroelectric and dielectric characterizations. (a) Ferroelectric hysteresis loops with PUND excitation pulse train, and (b) relative dielectric constant measured at room temperature in Pt/PZT/SRO capacitors on STO(001) and on STO-buffered Si(001) substrates. 

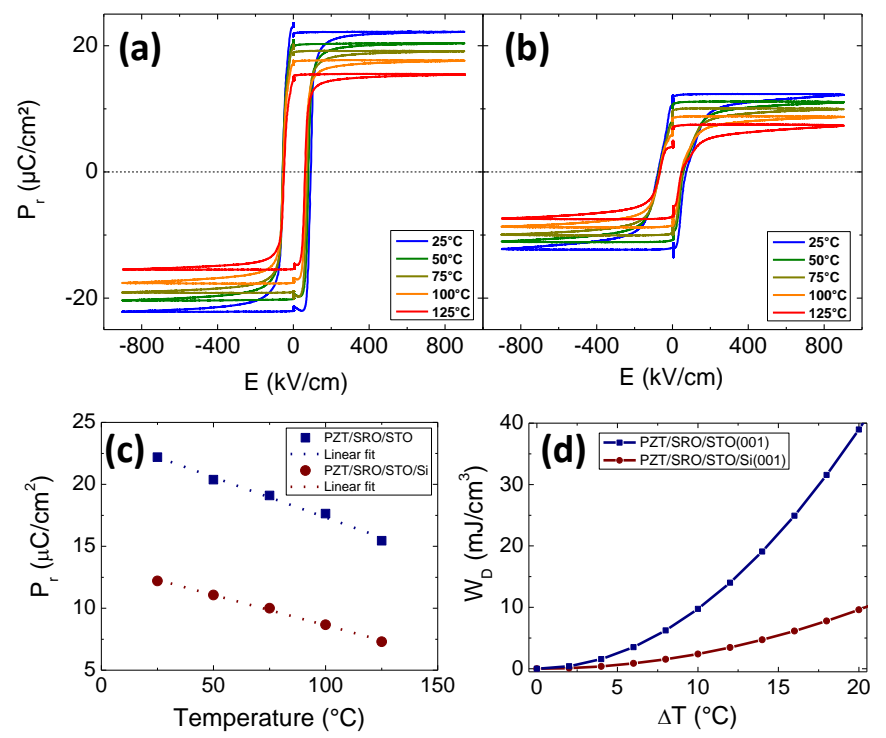

Fig. 5. Out-of-plane pyroelectric characterizations. (a-b) Ferroelectric hysteresis loops with PUND excitation pulse train measured at different stabilized temperatures on (a) heterostructure on STO(001) substrate and (b) STO-buffered $\mathrm{Si}(001)$ substrate. (c) Variation of the remnant polarization as a function of the temperature for both heterostructures. (d) Evaluation for both heterostructures of the converted pyroelectric energy density along the out-of-plane direction as a function of the temperature variation. 